Although EAE is a useful model for MS, clinical trials have shown that many efficacious therapies in the mouse have not worked in humans. Therefore, caution must be exercised in spite of the validation in mice of the two reported MS targets. However, this study does provide proof of principle for using microarray technology to identify new therapeutic targets. Databanks that contain large microarray data sets generated by these types of study will allow more meaningful cross-data-set comparisons to get the most out of the increasing numbers of such analyses.

Melanie Brazil, Associate Editor, Nature Reviews Drug Discovery

(2) References and links ORIGINAL RESEARCH PAPER Lock, C. et al. Gene-microarray analysis of multiple sclerosis lesions yields new targets validated in autoimmune encephalitis. Nature Med. 8, 500-507 (2002)

disease should fuel research for years to come.

Jane Alfred

(2) References and links ORIGINAL RESEARCH PAPER Giess, R. et al. Early onset of severe familial amyotrophic lateral sclerosis with a SOD-1 mutation: potential impact of CNTF as a candidate modifier gene. Am. J. Hum. Genet. $\mathbf{7 0}$, 1277-1286 (2002)

WEB SITE

NINDS amyotrophic lateral sclerosis

information page: http://www.ninds.nih.gov/ health_and_medical/pubs/als.htm

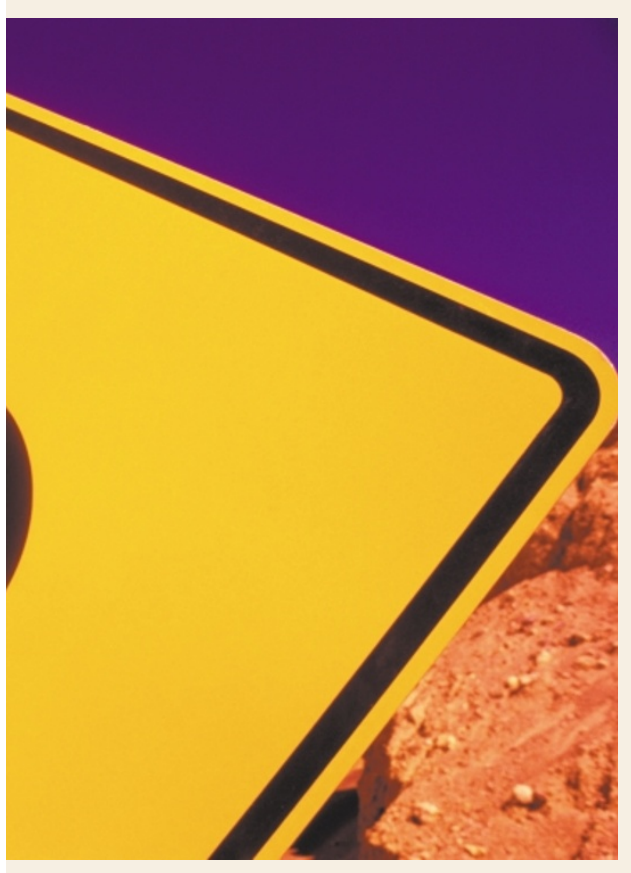

GENE EXPRESSION

\section{Peripheral oscillations}

Circadian rhythms are most often studied in association with behaviour and the underlying gene-expression changes in the brain. But circadian clocks that generate transcription periodicity also exist in many peripheral organs, as discussed in a new study published in Nature that demonstrates extensive circadian gene regulation in the hearts and livers of mice. In the biggest study of its kind, the authors show that this type of gene regulation is more extensive than previously thought and that, although it has a specialized role in a given tissue, it also has a broad biological function.

Although the fact that peripheral tissues have circadian regulation is not new, the extent of this regulation remained largely unknown. To investigate this, Storch et al. performed a microarray analysis on the hearts and livers of mice whose light/dark cycle had been synchronized. Using a set of genes with known circadian regulation as a guide, they found that $10 \%$ of liver genes and $8 \%$ of heart genes were regulated by the circadian clock. Although oscillations in gene expression were generally out of phase with each other in the liver, they were much more synchronized in the heart.

Interestingly, there was very little overlap between the genes that oscillate in the heart and in the liver - only 37 genes shared the same phase of peak expression. The authors point out that the core set of genes is important as it is likely to include those that are important for the functions of the peripheral clocks. Among them, they found genes involved in chromatin regulation, such as DNMT1-associated protein-1, and in ubiquitin pathways, suggesting that gene expression, in general, and protein stability are influenced by circadian rhythms.

To determine the kinds of processes that involve genes with circadian regulation, Storch and colleagues used a gene-ontology approach to sort the data into three categories: biological process, molecular function and cellular component. Their analysis indicated that, even in a single tissue, the circadian clock regulates many diverse processes, several of which are shared by the liver and the heart. So, the authors conclude that, although the genes regulated by circadian clocks are different in the two tissues, this type of regulation influences many related or overlapping processes.

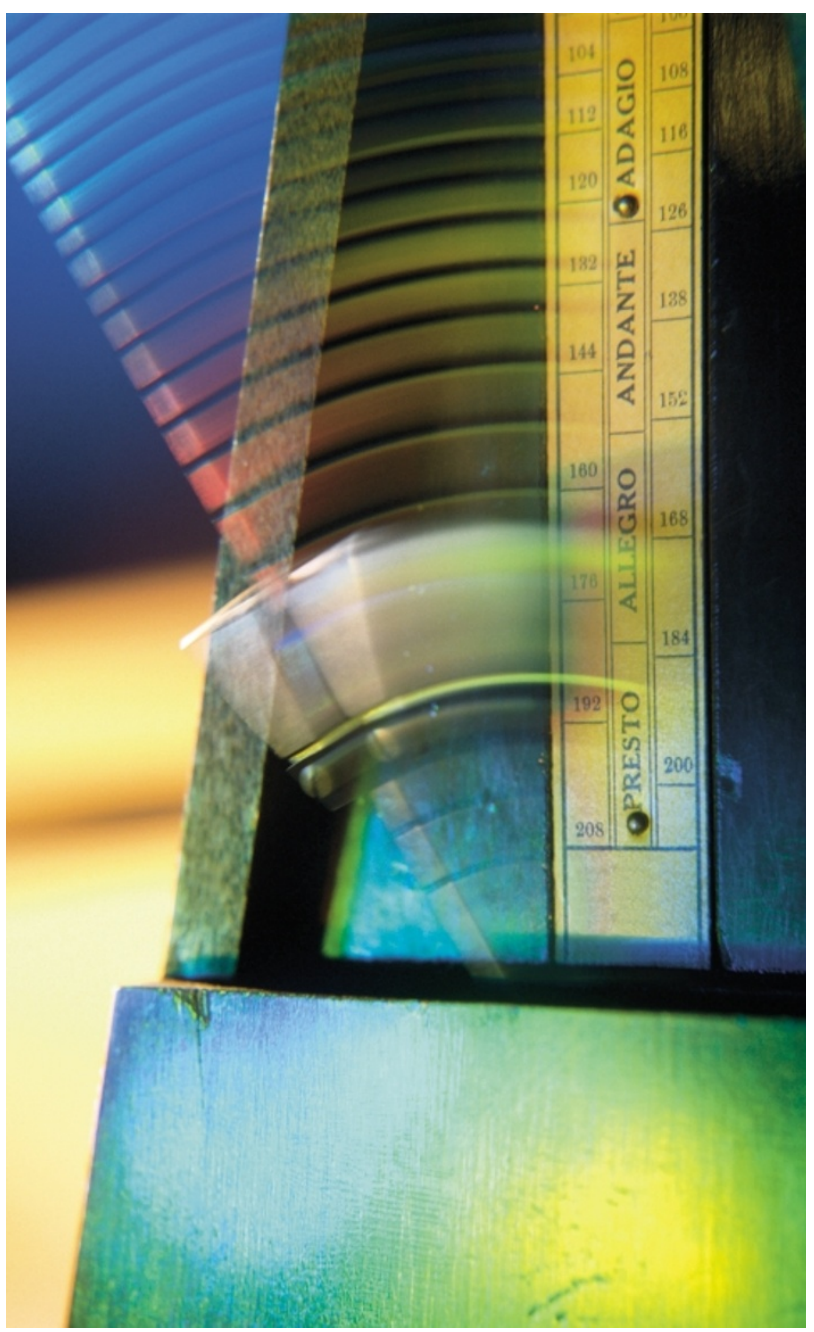

As the authors point out, there are some limitations to their study. They admit that transcript oscillations need not be reflected at the protein level, which depends, at least in part, on protein turnover. Also, for some proteins, circadian oscillations might have little physiological consequence. Nonetheless, together with another study published in Cell, which looked at circadian gene regulation in the liver and in a part of the hypothalamus that is known to harbour the 'pacemaker' of circadian control, this study substantially improves our understanding of circadian gene expression and provides evidence about the core clock mechanism that underlies it.

Magdalena Skipper

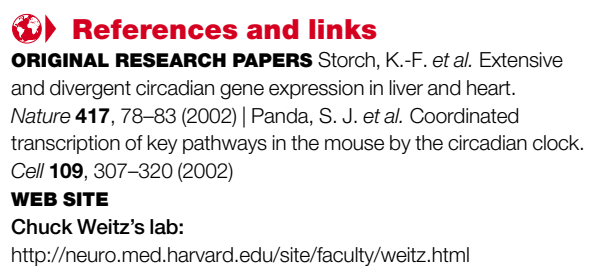

